\title{
Entropy Based k Nearest Neighbor Pattern Classification(EbkNN): En-route to Achieving a High Accuracy in Breast Cancer Diagnosis
}

\author{
Pushpam Kumar Sinha ${ }^{1}$ and Ankita Sinha ${ }^{2}$ \\ ${ }^{1}$ Department of Mechanical Engineering, Netaji Subhas Institute of Technology, \\ Amhara, Bihta, Patna, India \\ Email: pushpamsinhal [AT] gmail.com \\ ${ }^{2}$ Soft ware Engineer 2, Intuit IDC, \\ Bangalore, India \\ Email: ankitasinha0811 [AT] gmail.com
}

\begin{abstract}
Entropybased $k$-Nearest Neighbor pattern classification (EbkNN) is a variation of the conventional $k$-Nearest Neighbor rule of pattern classification, which exclusively optimizes the value of $k$-neighbors for each test data based on the calculations of entropy. The formula for entropy used in EbkNN is the one that has been defined popularlyin information theory for a set of $n$ different types of information (class) attached to a total of $m$ objects (data points) with each object defined by ffeatures. In EbkNN that value of $k$ is chosen for discrimination of given test data for which the entropy is the least non-zero value. Other rules of conventional kNN are retained in EbkNN. It is concluded that EbkNN works best for binary classification. It is computationally prohibitive to use EbkNN for discriminating the data points of the test dataset into number of classes greater than two. The bigg est advantage of EbkNN vis-à-vis the conventionalkNNis that in one single run of EbkNNalgorithm we get optimum classification of test data. But conventional $k N N$ algorithm has to be run separately for each of the selected range of values of $k$, and then the optimum $k$ to be chosen from amongst them. We also tested our EbkNN method on WDBC (Wisconsin Diagnostic Breast Cancer) dataset. There are 569 instances in this datasetand we made a random choice offirst 290 instances as training dataset and the rest 279 instances as test dataset. We got an exceptionally remarkable result with EbkNN method-accuracy close to 100\% and better than the ones got by most of the other researchers who worked on WDBC dataset.
\end{abstract}

Keywords - Binary classification, Euclidean distance, Training dataset, Information

\section{INTRODUCTION}

Pattern analy sis in which class is to be as signed to a given unknown data or test data based on the kno wn classes of training data, and in which no definite form of the mapping function (mapping class to the data) can be found, is most popularly referred to in the mathematical/statistical literature as nonparametric discrimination(NPD). $\mathrm{kNN}$ (k-Neare s $\mathrm{t}$ Neighbor) is the easiest, and yet powerful, machine learning algorithmfor NPD [1,2,3]. The seeds of the kNN were sown first in a technical report [4] by Evelyn Fix and J.L. Hodges, Jr. Suppose the classification is between two sets of population: one from data points $a_{1}, a_{2}, \ldots \ldots, a_{m}$ with probability density $x$ and the other from data points $b_{1}, b_{2}, \ldots \ldots, b_{n}$ with probability density $y$; the assigning of class to a new datapoint $\varepsilon$ depends on the likelihood ratio

$$
l(c)=x(c) / y(c)
$$

The problemarises with equation (1) when we do not exactly know $x, y$ but instead have to use their estimates. In [4] the authors sug gest severales timates of $x, y$ for different problems for both parametric and nonparametric discrimination, like, for example kernel density estimation. In the field of nonparametric discrimination, they were, however, the first in the world to give the nearest neighbor estimates of $x, y$, and thereby lay the foundation of kNN.

The work done on 1-NN (single nearest neighbor) and kNN in [4] was further extended in the works [5,6,7,8,9,10]. In [10], it was shown that for any sample size $n$, the single-Nearest Neighbor rule has a lower probability of error than the kNN for certain clas ses of dis tributions. [10] als o made it clear that there are two extremes of classification problem normally encountered, they are either parametric or nonparametric. The parametric problems are the ones in which the underlying statistical dis tribution of the observables are known. For the nonparametric problems certain common s ense 
based approaches may be made with regard to the decision rule, otherwise, strictly speaking there can be no optimal decision rule for this case. [10] states the lemma of the convergence of the nearest neighbor. Let $\left\{a_{1}, a_{2}, \ldots, a_{n}\right\}$ be the set of independent identically dis tributed randomvariables in the metric space $A$ classified into $M$ classes but with no underlying statistics. Let an unclassified randomvariable $a$ has as its closestneighbor $a_{m}$ formthe set $\left\{a_{1}, a_{2}, \ldots x, a_{n}\right\}$. Then $a_{m}$ tends to $a$ with probability one. This lemma holds for any metric, including the Euclidean, defined over the observables.

There can be several different types of distance metrics that can be computed amongst the data points of the training dataset and that between the query point and the data points of the training data, and the performance of the kNN classification depends on the way the distance metric is defined in particular application [11]. The distance metric most popularly used in the kNN classification is the Euclidean distance which treats the data points as vectors and neg lects any statis tical relationship that can be fitted to the clas sification example. However, it has been shown in several works that if the distance metric is trained to learn from the statistical distribution fitting the labelled data, there is an improvement in the performance of the kNN classifier many-folds [12,13,14]. [11] is an advancement over the works by $[12,13,14]$, and in this work the authors propose the learning Mahalanobis distance metric. The learning Mahalanobis distance metric is a linear transformation of the space of the training dataset in such a way that it bring s ab out two key changes in the conventional Euclidean distances calculated between the data points.

1. Firstly the large Euclidean distances between the two particular datapoints of the same class are op timized in the new metric to show a small distance

2. Secondly the small Euclidean dis tances between the two particular datapoints belonging to two different classes are magnified in the new metric to show a large distance

Making the dis tance metric learn this way ensures that the knearest neighbors of the unclassified data belong to the same class.

Before we move ahead with studying many more literature to introduce the problemdealt with in this work, let us first summarize the conventional kNN method as it is known today. Consider a typical NPD problem wherein $u$ is the unlabeled data to be assigned the class from the knowledge of training dataset of $n$ data points segregated into $m$ classes, each data point being defined by the value of $p$ features. Then the kNN method is all about finding knearest neighbors to $u$ from the training dataset for a certain fixed $\mathrm{k}$ which can be typically varied from 1 to $\sqrt{n}$ [1]. The neighbors are defined as those data points which are close to each other, the extent of closeness being quantified by the Euclidean distance(Ed) between the data points. TheEd between $u$ and the data point $v$ from the training dataset is calculated by the formula

$E d_{u-v}=\sqrt{\sum_{i=1}^{p}\left(f_{i u}-f_{i v}\right)^{2}}$

where $f_{i u}$ is the value of feature $i$ for unlabeled data $u$ and $f_{i v}$ is the value of feature $i$ for the data point $v$ from the training dataset. SuchEd is calculated between $u$ and each of the $n$ data points fromthe training dataset, denote th is set of Euclidean distances $\left\{E d_{u-v} \mid v=1,2, \ldots x, n\right\}$. For a chosen $\mathrm{k}$, the k data points from the training dataset corresponding to the $\mathrm{k}$ s mallest Euclidean distances fromthe set $\left\{E d_{u-v} \mid v=1,2, \ldots, n\right\}$ are grouped together. Then the class of the unlabeled data is the class of the majority of the datapoints in this group.

Despite its simplicity and power, the conventional $\mathrm{kNN}$ method posed two serious questions to the researchers over the years

1. The method is computationally expensive for large sample size and or many-dimensional features becau se one has to calculate the distances of the unlabeled data fromall the data points in the training dataset. Then how does one optimize the computational cost?

2. Which k should onechose forclas sification? This is obvious that as k is varied in any given particular NPD problem, the performance of the kNN too may vary. It is intuitive that the optimum $\mathrm{k}$ which has a higher probability of giving correct classification will vary from one situation to the other, like, for example, even between different unlabeled data points within the same NPD problem.

For achieving computational economy some of the works provided structure to the training dataset $[15,16,17,18,19,20,21]$. A few of the alternate approaches to achieve computationaleconomy, wherein the foc us is on reducing the size of the labeled dataset, are [3,22,23,24,25]. In [22], Hart proposed selecting the subset $\mathrm{S}$ from the whole training dataset $\mathrm{T}$ such that $1 \mathrm{NN}$ (1-Nearest Neighbor) with $\mathrm{S}$ discriminates the data points almost as accurately as $1 \mathrm{NN}$ does with $\mathrm{T}$. This way he removed the redundant data subsetfrom the whole training dat aset, and there by achieved computational economy. RNN (Reduced Nearest Neighbor rule) [23] is a refinement of CNN (Condensed Nearest Neighbor rule) [22], wherein the data points from the subset $\mathrm{S}$ of the whole training data set $\mathrm{T}$ selected by CNN are 
removed one by one to check whether the resulting reduced subset of subset $\mathrm{S}$ discriminates $\mathrm{T}$ correctly. [24] proposed selecting three very small training subsets out of the whole training data set, and then classifying the test data in each of these three training subsets by $1 \mathrm{NN}$ sub-classifier; the final clas sification being done by simple voting scheme to achieve a high accuracy. [25] did a careful study that given the training data set $\mathrm{T}$, which data points must be removed fro $\mathrm{m} T$ to get the subset $\mathrm{S}$ which discriminates $\mathrm{T}$ in a way so as not to affect the generalization accuracy and noise tolerance. They proposed 6 algorithms DROP1-DROP5 and DEL, DROP standing for Decremental Reduction Optimization Proce du re and DEL standing for Decremental Encoding Length. DROP1 is derived fromRNN [23] with the difference that once a data point is removed fromS to get new $S$ it is checked whether this reduced $S$ discriminates itself correctly rather than checking whether this reduced $\mathrm{S}$ discriminates $\mathrm{T}$ correctly.

Next we are concerned with the problemof optimizing $\mathrm{k}$ in $\mathrm{kNN}$. There are two ways to achieve the performance uniqueness in kNN: either get rid of $\mathrm{k}$ or fix $\mathrm{k}$ for each test or unlabeled data. The work that gets rid of $\mathrm{k}$ is by Sinha [26]; he calls the resulting method ANN(Alternative Nearest Neighbor). However, this (getting rid of k) is not the foc us of this paper. In this paper we discuss a new algorithmEbkNN (Entropy-based kNearest Neighbor), which we have found to optimize $\mathrm{k}$ for each given test data. But before we do that let us look at literature for some algorithms that optimizes $\mathrm{k}$ in kNN. [27] defines a new informative metric that tells how informative a particular data point from the training data set in the vicinity of unlabeled data is. The idea of informativeness is that highly informative points in the vicin ity of tes $t$ point have same class and are far from the points having dissimilar class. Ideally it is required that the informative metric be calculated for all the points in the training data set, but then the assignment of class to the test point based on informativeness will become computationally very expensive. So Song et al in [27] proposed two alg orithms of NPD based on informativeness: LI-kNN (Locally informative kNN) and GI-kNN (Globally informative kNN). In LI-kNN firs tly $\mathrm{k}$ nearest neighbors to the test point are chosen based on Euclidean dis tances and then out of these kneighbors the clas s of the most informative point is assigned to the test point. The GI-kNN differs from LI-kNN in that the neighbors are chosen not based on Euclidean distance but based on weighted Euclidean distance in an iterative algo rithm. [2] did bootstrap sampling of the training example and combined the technique with ne arest neighbor classifier and fou nd th at they got better performance than conventional kNN. [28] and [29] conducted text categorization studies with a variety of machine learning algorithms, one among thembeing kNN. They took kvalues solarge as 30,45 and 65; and found that the performance of the $\mathrm{kNN}$ on Reuters versions 3 and 4 was one of the best. There are a plethora of other literature that solves the problemof optimizing performance of $\mathrm{kNN}$ by fixing $\mathrm{k}[30,31,32,33]$. But because our method $(\mathrm{Eb} \mathrm{kNN})$ is not related in any way to either of these previous works, I merely make a mention of themhere and do not discuss them. Also because our work (EbkNN) is neither an advancement over these works [27,2,28,29,30,31,32,33] nor is derived from them, we do not immediately conclude or claim in this paper that EbkNN outperforms a subset of these and is inferior to the rest. Moreover, we do not state in this paper any mathematical theorem giving the lower and upper bounds on the probability of error of EbkNN. We have a strong belief that when NPD problems are to be solved by common sense, any amount of mathematical attempts to justify/rationalize the solution remain inert to the actual performance of the NPD method over real datasets. It is highly likely that what mathematics will infer may not reflect in actual application. So our main focus in this paper is to make the reader understand and appreciate EbkNN method exhaustively. One must take note of the simplicity and power of the method. Thereafter, we also apply the method to real breast cancer dataset and evaluate its performance. Our target is to achieve a high accuracy in breast cancer diagnosis by the EbkNN method. Let us see if we can do that. The breast cancer diagnosis is binary clas sification between whether the tumor is malignant or benign. Benign tumors are harmless and do notmetastasize upon the pas sage of time, whereas malignant tumors metastasize with the passage of time if left medically unattended to. Ideally we should have combined the features of either of the works $[3,22,23,24,25]$ into our method to take care of computational expenditure, but to keep things simple and to impart a better understanding of the EbkNN method to thereader we have not done so.

\section{THE EBKNN METHOD}

If there is a set of $n$ different types of information attached to a total of $m$ objects with each object defined by $f$ features/attributes, the entropy $s$ of this set of objects is defined mathematically in information theory [34] as

$$
s=-\sum_{\mathrm{i}=1}^{n} p_{\mathrm{i}} \log _{2} p_{\mathrm{i}}
$$

where $p_{\mathrm{i}}$ is the proportion of objects with information $i$. Interpreting the above information theory for the ty pical NPD problemin which the $m$ data points are clas sified into $n$ classes, the classes are the $n$ types of information and the data points are the $m$ objects. Hence the above equation for entropy holds for the stated typical NPD problem with $p_{i}$ being the proportion of data points of class $i$. 
The EbkNN method is all about selecting a single unique value of knearest neighbors to clas sify the query point, a nd as the name of the method suggests this is donebased on the calculation s of entropy. Just as we calculated entropy above for the entire training data set, we calculate entropy for each of the individual values of $\mathrm{k}$. So the fund amental ide a in EbkNN is that we chose that value of kfor classification for which theentropy is the least. However, this fu ndamental idea needs a little modification, given that for a certain $p_{i}=1$ (which means that all the datapoints grouped together for a certain $\mathrm{khas}$ same class), $s=0$. This means that for $\mathrm{k}=1$, definitely $s=0$. Therefore, the actual EbkNN method is that we choose that $k$ for classification for which the entropy is theleast non-zero value. Hence, 1-Nearest Neighbor decision rule is not integrated into EbkNN. The EbkNN method can be applied to classification of data points into any number of classes. But for the number of classes greater than 2, it is obvious that the method will be computationally very expensive. Hence in this paper we will be applying this method to binary classification only, as convenient simplifications can be done in computation.

In a binary clas sification problem let, out of $\mathrm{k}$ nearest neighbors, $q_{1}$ are the number of data points belon ging to class 1 , and $q_{2}$ are the number of data points belonging to class 2 . Note that, in this method, the neighbors are determined by calculating Euclidean dis tances- the conventional distance metric most popularly used in the kNNalgo rith $\mathrm{m}$. Then for this problem

$$
\begin{gathered}
p_{1}={ }^{q_{1}} /_{k} \\
p_{2}={ }^{q_{2}} / k=1-p_{1}
\end{gathered}
$$

By varying $q_{1}$ and $q_{2}$ over a range of natural numbers and doing calculation of entropy it is not hard to observe that as anyone s maller out of the two proportions $p_{1}$ and $p_{2}$ reduces the entropy reduces. In other words, the greater the deviation between $p_{1}$ and $p_{2}$, the smaller the entropy. For example consider the following two cases

Case 1: $p_{1}=0.15, p_{2}=0.85$

$\left|p_{1}-p_{2}\right|=0.7$

Let entropy for this case be $s_{1}$

Case 2: $p_{1}=0.9, p_{2}=0.1$

$\left|p_{1}-p_{2}\right|=0.8$

Let entropy for this case be $s_{2}$

It can be inferred immediately that $s_{2}<s_{1}$. This can be verified by calculation.

So, for binary clas sification problem, one need not explicitly calculate entropy for each value of k. Instead, that value of $\mathrm{k}$ be chosen for clas sification for which the smaller of the two proportions $p_{1}$ and $p_{2}$ is the leastamongst all $\mathrm{v}$ alu es of $\mathrm{k}$ considered. This is a very big simplification in computation for the EbkNN method. However, this liberty is not av ailable in EbkNN computations for the NPD problem with greater than two number of clas ses.

For $\mathrm{k}=m$, where $m$ is the sample size of the training dataset classified into two classes, an important question to ask is that ideally what should be $q_{1}$ and $q_{2}$ or $p_{1}$ and $p_{2}$ in the EbkNN method. Note that entropy is equal to 1 , the maxi mu m, for $p_{1}=p_{2}=0.5$. Hence we will choose the training dataset in a particular NPD problemin such a way that $m$ is even and $q_{1}=q_{2}=m / 2 . \mathrm{We}$ do this because then $\mathrm{k}=m$ will definitely not be the optimumk for clas sification.

Before we go ahead and apply the EbkNN method to the actual NPD problem, there is one last is sue that needs attention. What is the range of values of $\mathrm{k}$ for which we need to calculate entropy or the de viation $\left|p_{1}-p_{2}\right|$ ? $\mathrm{k}=1$ is definitely ruled out. $\mathrm{k}=2$ is als o ruled out because then $\left|p_{1}-p_{2}\right|$ is either 1 or 0 , i.e. entropy is either 0 or 1 respective ly . So at the lower end of the range we begin with that $\mathrm{k}>=3$ for which $\left|p_{1}-p_{2}\right| \neq 1$. Let us denote this value $k_{g}$. For the higherend of the range, suppose that at a particular instance $q_{1}>q_{2}$. Then $p_{1}={ }^{q_{1}} /\left(q_{1}+q_{2}\right)>p_{2}={ }^{q_{2}} /\left(q_{1}+q_{2}\right)$. Let the entropy at this instance be E. From this stage as $k=q_{1}+q_{2}$ is increased in increments of 1 , further suppose that $q_{1}$ remains static where as it is $q_{2}$ which increases in steps of 1 . As this happens entropy will first increase up to 1 when $q_{\text {2new }}=q_{1}, q_{\text {2new }}$ being the new value of $q_{2}$ after successive increments. Thereafter it (entropy) will reduce, and suppose that it becomes exactly equal to Efor $q_{\text {2new }}=q_{2}^{s}$. If $p_{2}^{s}$ be the new proportion of data points belonging to class 2 for new $k=q_{1}+q_{2}^{g}$, then

$$
\begin{gathered}
p_{2}^{s}=p_{1} \\
\text { or } q_{2}^{s} /\left(q_{1}+q_{2}^{s}\right)=q_{1} /\left(q_{1}+q_{2}\right) \\
\text { or } q_{2}^{s}=q_{1}^{2} / q_{2} \\
\text { If } q_{1}+q_{2}^{s}<m, \text { continue further }
\end{gathered}
$$


It is obvious that the instance $\mathrm{k}=q_{1}+q_{2}$ that Iam talking about above should be the one such that $\left|p_{1}-p_{2}\right|$ is maximum from amongst the subset of set $\{\mathrm{k} \mid \mathrm{k}=1,2, \ldots, \mathrm{m}\}$. For $m$ of the order of hundreds let us take this subset of length 10 , i.e., for every set of 10 ' $\mathrm{k}$ ' values beginning from the minimum $\mathrm{k}=k_{g}$ we find that $\mathrm{k}$ for which $\left|p_{1}-p_{2}\right|$ is maximum and check if $q_{1}+q_{2}^{s}<m$. The moment $q_{1}+q_{2}^{s} \geq m$, that will be the higher end of the range of $k$ for which we need to compareentropy or $\left|p_{1}-p_{2}\right|$. Once the optimized $\mathrm{k}$ is chosen this way the assigning of clas s to the unlabeled data is still done based on the majority voting of the classes of data points fromamongst this (optimized) $\mathrm{g}$ roup of $\mathrm{k}$ nearest neighbor data points.

\section{COMPUTER EXPERIMENTS WITH WDBC DATASET}

The real dataset on which we run the EbkNN and the kNN method in this work is the WDBC(W is consin Diagn ostic Breast Cancer) dataset fromthe UCI machine learning repository. Dr. William H. Wolberg, W. Nick Street, an d Olvi L. Mangasarian are the creators of this dataset. There are 569 data points in this dataset, and 32 columns. The first column is the patient ID. The second column is diagnosis: $\mathrm{B}$ for Benign and $\mathrm{M}$ for malignant. The rest 30 columns have the values of 30 features. However, effectively there are only 10 basic features - radius, texture, perimeter, area, smoothness, compactness, concavity, concave points, symmetry, and fractal dimension. Actually what are these $10 \mathrm{~b}$ as ic fe at ures ? During the biopsy procedure to detect whether the abnormal lump or mass of cells formed in the breast is tumoror not, a fine needle aspirate of few numbers of cells from the lump or mass is taken and observed und er mic ros cope. During microscopic examination meas urements of these 10 basic features of the cell are made. Columns 3 to 12 (in the W DBC dataset) are res pectively the mean values of these 10 features, columns 13 to 22 are respectively the standard error (s .e.) of these 10 features and columns 23 to 32 are respectively the worst values of these 10 features. Everyone knows what is meant by mean, so I should not define it here. Standard error (s.e.) is given by

$s_{x} e_{x}=\sigma / \sqrt{n}$

where $\sigma$ is standard deviation and $n$ is number of sample points in the data. It is actually the difference between accurate mean and the observed mean. Worst is the mean of three of the largest values of concerned feature. Stand ard error by itself has no meaning; specially it is highly likely that it has directly no role to play in the diagnosis. But with its effect included in mean, it will surely have role to play in the diagnosis. So now theimportant question-How do we inclu de the effect of standard error in mean ? We add the mean of a particular feature say feature1_mean to the standard error of that feature say feature1_se to get the upper limit on the value of feature say feature1_ul. Similarly we subtract from the mean of a particularfeature say feature1_mean the standard error of that feature say feature1_se to get the lower limit on the value of feature say feature1_ll. Mathematically

feature1_ul=feature1_mean +feature1_se

feature1_ll=feature1_mean - feature1_se

We did so forall the basic 10 features mentioned above, and created a new data file "processed_data.csv". Colu mn 1 of this file is index beginning from 0 , column 2 is patient ID, column 3 is diagnosis, columns 4 to 13 is the mean of the 10 features mentioned above, columns 14 to 23 is the standard error of the 10 features mentioned above, columns 24 to 33 is the worst values of the 10 features mentioned above, column 34 is blank, columns 35 to 44 is the upper limit of the 10 features mentioned above, and columns 45 to 54 is the lower limit of the 10 features mentioned above. While calculating the Euclidean distances, both for EbkNN and kNN, we considered only the features in columns 24 to 33, colu mn s 35 to 44, and columns 45 to 54 .

While selecting the training subset out of a set of 569 instances, we could have used the results of either of the works $[3,22,23,24,25]$. But we did something else. Because 569 is a big number we considered that the training subset so larg e as a little over $50 \%$ of the whole set should be sufficient to capture almost all pos sible variations of values of features. Then we will run EbkNN and kNN over this subset as training data and the rest as test data. In next step, whatever mis classifications we get in the first step will be removed from the test data and included in the training data. This sounds somewhat like [22], but is far different from it. The major difference being that the training subsetis chosen randomly in this work, given the large number of instances.

First 290 instances ( 145 benign and 145 malignant instances) are chosen as training data and the rest 279 instances are test data. We call it experiment 1 . What performance meas ure is the most important in diagnosis of dis ease ? It is accuracy and the accuracy of any diagnostic tool should ideally be $100 \%$. Hence, in this work too the only performance measure we will be interested in is accuracy. And our aimin this work will be to take accuracy as close as possible to $100 \%$. 


\section{RESULTS}

\subsection{Experiment 1}

The accuracy of EbkNN method $=92.1147 \%$. The optimized value of $k$ for EbkNN method which we compute for as signing clas s to the test datapoint by majority voting varied fromso small as 5 to as large as 98 , the results of all 279 test datapoints considered. The accuracy of $\mathrm{kNN}(\mathrm{k}=5)$ method $=92.1147 \%$

\subsection{Experiment 2}

The misclassified data points in EbkNN for experiment 1 are data point nos. 298, 299, 341, 348, 364, 376, 380, 386, 407, 414, 431, 449, 466, 473, 477, 482, 492, 509, 514, 533, 537, and 542.

The misclassified data points in kNN for experiment 1 are data point nos. 298, 299, 341, 348, 364, 376, 380, 386, 407, 422, 431, 466, 473, 477, 482, 492, 509, 514, 519, 533, 537, and 542.

We now include these misclassifications in training data and remove themfromthe test dataset. So, for both EbkNN an d $\mathrm{kNN}$, the training data has 312 data points and the test data has 257 data points. Note that in EbkNN method for experiment 2 the number of benign samples $(=162)$ and the number of malignant samples $(=150)$ are not equal. So, a naturalquestion to as $k$ is that if this skew can cause the optimumk to be 312 for any one particular test $d$ ata. Let $u$ s convince ourselves that this will never be the case here before we go ahead. The biggest $\left|p_{1}-p_{2}\right|$ (for meaning of $p_{1}$ and $p_{2}$, refer section 2) can be as small as 0.34 if optimum $\mathrm{k}=3$ in the application of EbkNN method to any dataset, which is definitely greater than $\left|p_{1}-p_{2}\right|$ value $(=12 / 312=0.03846)$ for optimum $\mathrm{k}=312$ in the application of EbkNN method to this dataset here in experiment 2 .

The accuracy of EbkNN method $=98.8327 \%$ ( 3 errors fromamongst 257 test data points)

The accuracy of $\mathrm{kNN}(\mathrm{k}=5)$ method $=98.0545 \%$ (5 errors fromamongst 257 test datapoints)

\subsection{Experiment 3}

If there are large number of features defining the datapoints of a given dataset, we can combine these features in a manner so that each combined component (called the principal component) is orthogonal to every other combined component. This process of feature extraction or dimensionality reduction is called Principal Component Analy sis (PCA) [1]. We will not go into the details of PCA as this is not the focus of this paper. But the reas on that Iam talking ab out it here is that we also did PCA of the data file "processed data.csv" with entries in columns 24 to 33,35 to 44 , and 45 to 54; and the number of principal components into which the features were extracted were 5,10 and 15 respectively. We thereafter chose first 290 instances of "processed_data.csv" as training data and the rest 279 instances as te st data and classified the test data with the application of the $\bar{E} b k N N$ method. Then we also included the misclassifie d te st $d$ ata in training dataset (as in experiment 2) and ran the EbkNN method again. The accuracies that we obtained for all the three PCA components 5, 10 and 15, were far less than that obtained in experiment 2 . Hence we do not make a mention of the results of this experiment here.

\section{CONCLUSIONS}

The accuracies of the EbkNN method and $\mathrm{kNN}(\mathrm{k}=5)$ method are same for experiment 1, where as in experiment 2 the accuracy of the EbkNN method was better than that of the $\mathrm{kNN}(\mathrm{k}=5)$. This means that in going from exp eriment 1 to experiment 2 there was no over-fitting of the data in EbkNN; EbkNN showed better noise tolerance than kNN. It is possible that forboth experiment 1 and experiment 2 , the $\mathrm{kNN}$ method may performthe best for kother than 5 . But for this we have to run the $\mathrm{kNN}$ algorithmseparately for a range of values of $\mathrm{k}$. This is the dis advantage of the convention al $\mathrm{kNN}$ algorithm, which we have overcome in ournewly found EbkNN method. In single run of the EbkNN meth od we pick the optimumk separately and exclusively for each test data. Anyhow we already found a high enough accuracy, as close to $100 \%$ (98.8327\%) as pos sible, with the EbkNN method. This accuracy is the best fromamong st the works of other researchers on WDBC dataset $[35,36,37,38,39,40,41,42,43]$ that the authors of this paper know of. The work by [43] is the closest that it gets to our work. Their best accuracy is $98.62 \%$ for $\mathrm{kNN}$ with chi-square based feature selection. We are sad that we failed to further improve our accuracy of $98.8327 \%$ with PCA. PCA seldomfails and it is amazing that PCA failed here.

It is not so that we did not do feature selection/extraction in experiment 1 and experiment 2. Calculating the up per limit values and the lower limit values of the 10 basic effective features in WDBC dataset was a part of the process of feature extraction only. No other researcher till now has done so with the WDBC dataset and we proudly claim that we are the first one in world to do so, and it reaped fruits for us. The feature extraction that we did in experiment 1 and 
experiment 2, however, was not the one which reduced number of features. Why the EbkNN method gave such promising result with the WDBCdataset is a matter for investigation by mathematicians and statis ticians alike.

It was serendipity that we dis covered high accuracy of the EbkNN method in breast cancerd iag nosis. Howe ver, it does not follow that the EbkNN method will demonstrate similar performance for other datasets. In fact, the performance of any machine learning algorithmover any dataset is dependent on the internal statistical structure of the dataset. Hence for different datasets the machine learning alg orithm which shows the best performance will be different.

\section{REFERENCES}

[1] S. Dutt, S. Chandramouliand A.K. Das. Machine Learning. Pearson India Education Services Pvt Ltd. Noida. 2020.

[2] Y. Hamamoto, S. Uchimura, and S. Tomita, "A Bootstrap Techniquefor Nearest Neighbor Clas sifier Design," IEEE TRANSACTIONS ON PATTERN ANALYSIS AND MACHINEINTELLIGENCE, vol. 19, no. 1, pp. 73-79, 1997.

[3] E. Alpaydin, "Voting Over Multiple Condensed Nearest Neoghbors," Artificial Intelligence Re view, vol. 11, p p. 115-132, 1997.

[4] E. Fix and J. Hodges. Discriminatory Analysis: Nonparametric Discrimination: Consistency Properties. USAF School of Aviation Medicine, Report No 4, 1951.

[5] M. V. Johns, '(An empirical Bayes approach to non-parametric two-way clas sification," in Studies in Item Analysis and Predic-tion, H. Solomon, Ed. Stanford, Calif.: Stanford University Press, 1961.

[6] L. N. Kanal, "Statistical methods for pattern classification," Philco Rept., 1963; originally appeared in T. Harley et al, "Semi-automatic imagery screening research study and expen-mental investigation," Philco Rep orts, VO43-2 and VO43-3, Vol. I, sec.6, and AppendixH, prepared for U. S. Army Electronics Research and Development, Lab. under Contract DA-36-039-SC-90742. March 29. 1963.

[7] G. Sebestyen, Decision Making Processes in Pattern Recognition. New York: Macmillan, 1962, pp. 90-92.

[8] Nils Nils son, Learning Machines. New York: McGraw-Hill, 1965, pp. 120-121. D. 0.

[9] Loftsgaarden and C. P. Ques enberry, "A nonparametric estimate of a multivariate density function," A nnals Math Stat., vol. 36, pp. 1049-1051, June 1965.

[10] T. M. Cover and P. E. Hart, "Nearest Neighbor Pattern Clas sification," IEEE Trans. Inform. Theory, vol. IT-13, pp . 2127, 1967.

[11] K. Q. Weinberger and L. K. Saul, "Distance Metric Learning for Large Margin Nearest NeighborClas sific ation," Journal of Machine Learning Research, vol. 10, pp. 207-244, 2009.

[12] S. Chopra, R. Hadsell, and Y. LeCun," Learning a similiarty metric discriminatively, with application to face verification,".In Proceedings of the IEEE Conference on Computer Vision and Pattern Rec ognition (CVPR-05), pages 349-356, San Diego, CA, 2005

[13] J. Goldberger, S. Roweis, G. Hinton, and R. Salakhutdinov,"Neighbourhood components analysis," In L. K. Saul, Y. Weiss, and L. Bottou, editors, Advances in Neural Information Processing Systems 17, pag es 513-520, Cambridg e, MA, 2005. MIT Press.

[14] S. Shalev-Shwartz, Y. Singer, and A. Y. Ng," Online and batch learning of ps eudo-metrics," In Proceedings of the Twenty First International Conference on Machine Learning (ICML-04), pages 94-101, Banff, Canada, 2004.

[15] J.K. Uhlmann ," Satis fying general proximity/s imilarity queries with metric trees," Information Processing Letters; 40: 175-179, 1991.

[16] T. Liu, A.W. Moore and A. Gray,"New Algorithms for Efficient High-Dimensional Nonparametric Clas sification," Journal of Machine Learning Research; 7: 1135-1158, 2006.

[17] R.F. Sproull," Refinements to Nearest Neighbor Searching in k-Dimensional Trees," Algorithmica; 6:579-589, 1991.

[18] S.Z. Li, K.L. Chan and C. Wang," Performance Evaluation of the NFL Method in Image Classification and Retrieval," IEEE Trans on Pattern Analysis and Machine Intelligence; Vol22-Is sue 11,2000.

[19] Y. Zhou and C. Zhang, "Tunable Nearest Neighbor Clas s," Pattern Recognition ;346-349 pp, 2007.

[20] Y.C. Liaw, C.M. Wu and M.L. Leou," Fast Exact k Nearest Neighbors Search using an Orthogonal Search Tree," Pattern Recognition; Vol43-Is sue 6:2351-2358, 2010.

[21] J. McNames," Fast Nearest Neighbor Algorithm based on Principal Axis Search Tree," IEEE Trans on Pattern Analysis and Machine Intelligence; Vol23-Issue 9:964-976, 2001.

[22] P. Hart, "The Condensed Nearest Neighbour Rule," IEEE Transactions on Information Theory, vol. 14, pp. 515-516, 1968.

[23] G. Gates, "The Reduced Nearest Neighbour Rule," IEEE Transactions on Information Theory, vol. 18, pp. 431 -433, 1972. 
[24] M. Kubat and M. Jr, "Voting Nearest-Neighbour Subclassifiers," in Proceedings of the 17th International Conference on Machine Learning, ICML-2000, Stanford,CA, pp.503-510.,2000.

[25] D. R. Wilson and T. R. Martinez, "Reduction Techniques for Instance-Based Learning Algorithms," Machine learning, vol. 38, no. 3, pp. 257-286, 2000.

[26] P. K. Sinha, "Modifying one of themachine learning algorithms kNN to make it independent of the parameter $\mathrm{k}$ by re-defining neighbor," I.J. Mathematical Sciences and Computing, 4, 12-25, 2020.

[27] Y. Song, J. Huang, D. Zhou, H. Zha, and C. L. Giles, "Iknn: Informative k-nearest neighbor pattern classific ation," in Proceedings of the 11th European conference on Principles and Practice of Knowledge Dis covery in Datab ases, Berlin, pp. 248-264., 2007

[28] Y. Yang, "An evaluation of statistical approaches to text categ orization," Information Retrieval, vol. 1, pp. 69-90, 1999.

[29] Y. Yang and X. Liu, "A re-examination of text categorization methods," in Proceedings of SIGIR-99, 22nd ACM International Conference on Research and Development in Information Retrieval, Berkeley, pp. 42-49, 1999.

[30] M. Jirina and M. J. Jirina, "Classifier Based on Inverted Indexes of Neighbors," Institute of Computer Science, Technical Report No. V-1034, 2008.

[31] M. Jirina and M. J. Jirina, "Using Singularity Exponent in Distance Based Clas sifier," in Proceedings of the 10th International Conference on Intelligent Sy stems Design and Applications (ISDA2010), Cairo, pp. 220-224, 2010.

[32] M. Jirina and M. J. Jirina, "Classifiers Based on In verted Distances," in New Fundamental Technologies in Dat a Mining, K. Funatsu, Ed. InTech, vol. 1, ch. 19, pp. 369-387, 2011.

[33] A. B. Hass anat, M. A. Abbadi, and G. A. Altarawneh, "Solving the problemofthe K parameter in KNN clas sifier using an Ensemble Learning Approach," International Journal of Computer Scienceand Information Security, Vol. 12, No. 8, pp.33-39, 2014.

[34] C.E. Shannon, "A mathematical theory of communication," Bell SystemTechnical Journal, 27(3): 379-423, 1948.

[35] S. Srivastava, N. Sharma, S.K. Singh, and R. Srivastava," Quantitative analysis of a general frame work of a CAD tool for breast cancer detection frommammograms, "J Med Imaging Health Inform, 4, 654-74, 2014.

[36] S. Saini, and R. Vijay," Mammogram analysis using feedforward back propag ation and cascade-forward back propagation artificial neural network," In 2015 Fifth International Conference on Communication Systems and Network Technologies IEEE, pp 1177-80, 2015.

[37] M.M. Pawar, and S.N. Talbar," Genetic fuzzy system (GFS) based wavelet co-occurrence feature selection in mammogram classification for breast cancer diagnosis," Perspect Sci, 8, 247-50, 2016.

[38] S.J.S. Gardezi, I. Faye, F. Adjed, N. Kamel, and M.M. Eltoukhy," Mammogramclas sification using curvelet GLCM texture features and GIST features," In International Conference on Advanced Intelligent Systems and In formatics Springer Cham, pp 705-13, 2016.

[39] K. Vaidehi, and T.S. Subashini,"Automatic characterization ofbenign and malignant masses in mammo graphy," Procedia Comput Sci, 46, 1762-9, 2015

[40] J. Harefa , A. Alexander, and M. Pratiwi," Comparison classifier: support vector machine (SVM) and K-nearest neighbor (K-NN) in digital mammogram images,"Jurnal Informatika dan Sistem Informasi, 2, 35-40, 2017.

[41] M. Pratiwi, J. Harefa, and S. Nanda,"Mammograms classification using gray-level co-occurrence matrix and ra dial basis function neural network," Procedia Comput Sci, 59, 83-91, 2015.

[42] H. Rajaguru, S. Chakravarty S.R., "Analysis of Decis ion tree and k-nearest Neighbor algorithmin the clas sification of breast cancer,” A sian Pac J Cancer Prev, 20 (12), 3777-3781, 2019.

[43] Z. Mushtaq, A. Yaqub, S. Sani \& Adnan Khalid," Effective K-nearest neighborclas sifications for Wisconsin breast cancer data sets," Journal of the Chinese Institute of Engineers, pp 1-13, DOI: 10.1080/02533839.2019.1676658, 2019. 